\title{
On the stability of the BEK family of rotating boundary-layer flows for power-law fluids
}

\author{
M. A. Abdulameer ${ }^{\mathrm{a}}$, P. T. Griffiths ${ }^{\mathrm{b}}$, B. Alveroğlu ${ }^{\mathrm{a}}$, S. J. Garrettt,* \\ ${ }^{a}$ Department of Mathematics, University of Leicester, Leicester, LE1 7RH, UK \\ ${ }^{b}$ Department of Mechanical Engineering and Mathematical Sciences, Oxford Brookes University, Oxford, OX33 \\ $1 H X, U K$ \\ ${ }^{c}$ Department of Engineering, University of Leicester, Leicester, LE1 7RH, UK
}

\begin{abstract}
We consider the convective instability of the BEK family of rotating boundary-layer flows for shear-thinning power-law fluids. The Bödewadt, Ekman and von Kármán flows are particular cases within this family. A linear stability analysis is conducted using a Chebyshev polynomial method in order to investigate the effect of shear-thinning fluids on the convective type I (inviscid crossflow) and type II (viscous streamline curvature) modes of instability. The results reveal that an increase in shear-thinning has a universal stabilising effect across the entire BEK family. Our results are presented in terms of neutral curves, growth rates and an analysis of the energy balance. The newly-derived governing equations for both the steady mean flow and unsteady perturbation equations are given in full.

Keywords: von Kármán flow, Ekman layer, Bödewadt flow, Power-law fluid, Convective instability, Flow control
\end{abstract}

\section{Introduction}

There has been significant interest in the stability and transition of the three-dimensional boundary-layer flow due to the rotating disk (that is the von Kármán [1] flow) in recent decades. The seminal study of the stability properties of the Newtonian rotating-disk boundary layer was performed by Gregory et al. [2], and there the first experimental observation of stationary crossflow vortices and the first theoretical stability analysis are presented. Some years later, Malik [3], utilsing the parallel-flow approximation, extended Gregory et al.'s high-Reynoldsnumber analytics and computed the neutral curves for stationary disturbances. Malik identified two distinct instability modes. The first mode (denoted type I), due to inviscid crossflow instability, was shown to be the dominant mode and was associated with Gregory et al.'s prior

\footnotetext{
${ }^{*}$ Corresponding author

Email address: stephen.garrett@le.ac.uk (S. J. Garrett)
} 
results. The additional second mode (denoted type II) was shown to be viscous in nature and attributed to external streamline curvature and Coriolis forces. In the same year, Hall [4] approached the problem rigorously and presented a high-Reynolds-number linear asymptotic analysis. Complete agreement between Hall and Malik's studies is found in the appropriate parameter limit.

Following these important milestones the seemingly simple system has continued to attract attention and it remains under active investigation to this day. The interested reader is referred to the literature for full information on the latest developments concerning the transition to turbulence via the generation of a non-linear global mode (see, for example, $[5,6,7,8]$ ).

This current paper is concerned with the stability characteristics of the family of boundarylayer flows attributed to a differential rotation rate between a lower disk and upper fluid in rigid-body rotation. Particular arrangements of this dual rotating system include the von Kármán [1], Ekman [9], and Bödewadt [10] boundary-layer flows. The von Kármán boundary layer arises when the lower disk rotates under a stationary fluid, the Ekman layer occurs when the disk and fluid rotate with approximately the same angular velocity, and the Bödewadt layer occurs when the fluid rotates above a stationary disk. There is a continuum of intermediate cases between these standard configurations and collectively these form the BEK family.

The mean-flow solutions of the entire BEK family for Newtonian flows are well understood $[11,12,13]$. In contrast, the stability characteristics of this family of flows has received relatively minimal attention, motivated largely by a desire to simply generalise the active research on the rotating-disk (i.e. von Kármán) system. In particular, Lingwood [14] presents local convective and absolute instability analyses of the Newtonian boundary layer and concludes that the limiting case of the rotating disk is the most stable configuration within the family. More recently, Lingwood and Garrett [15] discuss the use of mass flux through the lower disk as a potential flow-control mechanism. Various experimental studies concerning the stability, transition and control of these types of flows has been an area of more recent active research $[16,17,18]$.

It is our intention here to generalise Lingwood's original work in this area to incorporate the effects of non-Newtonian fluids. Our motivation is to explore the potential for using such fluids to optimise the performance of rotor-stator systems in engineering applications.

With regards to prior studies of the non-Newtonian boundary-layer flow over a rotating disk, Mitschka and Ulbrecht [19] were the first to extend the von Kármán similarity solution to incorporate fluids that adhere to a power-law governing viscosity relationship. That study, involving both shear-thickening and shear-thinning fluids, was later verified by Andersson et al. 
[20] in order to test the reliability of their numerical solutions. However, further to this, Denier and Hewitt [21] readdressed the problem showing that asymptotic matching considerations need to be taken in to account in order to able to accurately describe the flow of shear-thinning power-law fluids. In the shear-thickening regime it transpires that the boundary-layer solutions are complicated by a region of zero viscosity away from the wall. For these reasons, in this study, we will restrict our attention to moderately shear-thinning fluids only. For full details regarding the asymptotic structure of the solutions the interested reader is refereed to Denier and Hewitt [21].

Much more recently Griffiths et al. [22] considered a rigorous asymptotic stability analysis of the shear-thinning boundary-layer flow over a rotating disk. This work was then extended by the same authors Griffiths et al. [23] to compute the neutral curves of convective instability (working under the parallel-flow assumption) and complete agreement was found with their prior asymptotic analysis. These two papers can be considered as the non-Newtonian generalisations of Hall [4] and Malik [3], respectively. Griffiths [24] later extends the power-law studies to include the Bingham [25] and Carreau [26] models of non-Newtonian viscosity. He finds that a generalisation of the von Kármán similarity solution is applicable for a variety of different inelastic and viscoplastic non-Newtonian models.

In this current paper we extend the non-Newtonian, inelastic study of Griffiths et al. [23], to the entire BEK family of rotating boundary-layer flows. A Chebyshev polynomial method is used to consider the effects shear-thinning power-law fluids have on the type I and type II modes of instability.

This paper proceeds as follows: In Section 2 the steady boundary-layer flows of the BEK system for fluids with a governing viscosity relationship adhering to a power-law model are formulated and the profiles presented. A local convective instability analysis is presented in Section 3 and the neutral curves, critical Reynolds numbers, and convective growth rates are detailed for a variety of flow parameters. An energy-balance analysis is considered in Section 4 and finally our conclusions are drawn in Section 5. All newly-derived equations are presented in detail where appropriate in our discussion.

\section{Formulation}

We consider a family of incompressible, shear-thinning boundary-layer flows above an infinite rotating disk located at $z^{*}=0$. Distinct flows within this family are generated by a differential rotation rate between this solid boundary and a fluid in rigid-body rotation (see 
$[14,15])$. Particular cases within the family are the Bödewadt, Ekman and von Kármán boundary layer flows and we denote the entire family as the BEK system. Both rotating components (disk and fluid) are assumed to rotate in the same direction and about the same vertical axis with angular velocities $\Omega_{D}^{*}$ and $\Omega_{F}^{*}$, respectively. The von Kármán layer appears when the fluid is stationary and the disk rotates, i.e., $\Omega_{F}^{*}=0$ and $\Omega_{D}^{*} \neq 0$; the Ekman layer is such that $\Omega_{F}^{*} \approx \Omega_{D}^{*}$; and the Bödewadt is such that $\Omega_{F}^{*} \neq 0$ and $\Omega_{D}^{*}=0$. Furthermore, there exists a continuum of cases between these particular examples in which both the disk and fluid rotate with different angular velocities.

The continuity and Navier-Stokes equations in a frame of reference rotating with the lower disk, at fixed angular velocity, are expressed as follows

$$
\begin{aligned}
\boldsymbol{\nabla} \cdot \boldsymbol{u}^{*} & =0, \\
\frac{\partial \boldsymbol{u}^{*}}{\partial t^{*}}+\boldsymbol{u}^{*} \cdot \boldsymbol{\nabla} \boldsymbol{u}^{*}+\boldsymbol{\Omega}^{*} \times\left(\boldsymbol{\Omega}^{*} \times \boldsymbol{r}^{*}\right)+2 \boldsymbol{\Omega}^{*} \times \boldsymbol{u}^{*} & =-\frac{1}{\rho^{*}} \nabla p^{*}+\frac{1}{\rho^{*}} \boldsymbol{\nabla} \cdot \boldsymbol{\tau}^{*} .
\end{aligned}
$$

Here $\boldsymbol{u}^{*}=\left(U^{*}, V^{*}, W^{*}\right)$ are the velocity components in cylindrical polar coordinates $\left(r^{*}, \theta, z^{*}\right)$, $t^{*}$ is time, $\Omega^{*}=\left(0,0, \Omega^{*}\right)$ and $\boldsymbol{r}^{*}=\left(r^{*}, 0, z^{*}\right)$. The fluid density is $\rho^{*}$ and $p^{*}$ is the fluid pressure.

The stress tensor $\boldsymbol{\tau}^{*}$ for generalised Newtonian models, such as the power-law model, is defined by

$$
\tau^{*}=\mu^{*} \dot{\gamma}^{*} \quad \text { with } \quad \mu^{*}=\mu^{*}\left(\dot{\gamma}^{*}\right)
$$

where $\dot{\gamma}^{*}=\boldsymbol{\nabla} \boldsymbol{u}^{*}+\left(\boldsymbol{\nabla} \boldsymbol{u}^{*}\right)^{\mathrm{T}}$ is the rate of strain tensor and $\mu^{*}\left(\dot{\gamma}^{*}\right)$ is the non-Newtonian viscosity. The magnitude of the rate of strain tensor is given by

$$
\dot{\gamma}^{*}=\sqrt{\frac{\dot{\gamma}^{*}: \dot{\gamma}^{*}}{2}} .
$$

For power-law fluids the governing relationship for $\mu^{*}\left(\dot{\gamma}^{*}\right)$ is

$$
\mu^{*}\left(\dot{\gamma}^{*}\right)=m^{*}\left(\dot{\gamma}^{*}\right)^{n-1}
$$

where $m^{*}$ is the consistency coefficient and $n$ is the dimensionless power-law index. For $n<1$ we have a pseudoplastic fluid where the viscosity decreases with increased rate of strain (i.e., shear thinning). For $n>1$ we have a dilitant fluid where the viscosity increases with increased rate of strain (i.e., shear thickening). The classical Newtonian viscosity law is recovered for the particular parameter value $n=1$.

The governing boundary-layer equations are formulated in a frame rotating with the lower disk, i.e., at $\Omega_{D}^{*}$, and are expressed in cylindrical-polar coordinates $\left(r^{*}, \theta, z^{*}\right)$ as

$$
\frac{1}{r^{*}} \frac{\partial\left(r^{*} U_{0}^{*}\right)}{\partial r^{*}}+\frac{1}{r^{*}} \frac{\partial V_{0}^{*}}{\partial \theta}+\frac{\partial W_{0}^{*}}{\partial z^{*}}=0
$$




$$
\begin{gathered}
\frac{\partial U_{0}^{*}}{\partial t^{*}}+U_{0}^{*} \frac{\partial U_{0}^{*}}{\partial r^{*}}+\frac{V_{0}^{*}}{r^{*}} \frac{\partial U_{0}^{*}}{\partial \theta}+W_{0}^{*} \frac{\partial U_{0}^{*}}{\partial z^{*}}-\frac{\left(V_{0}^{*}+r^{*} \Omega_{D}^{*}\right)^{2}}{r^{*}}=\frac{1}{\rho^{*}} \frac{\partial}{\partial z^{*}}\left(\mu_{0}^{*} \frac{\partial U_{0}^{*}}{\partial z^{*}}\right), \\
\frac{\partial V_{0}^{*}}{\partial t^{*}}+U_{0}^{*} \frac{\partial V_{0}^{*}}{\partial r^{*}}+\frac{V_{0}^{*}}{r^{*}} \frac{\partial V_{0}^{*}}{\partial \theta}+W_{0}^{*} \frac{\partial V_{0}^{*}}{\partial z^{*}}+\frac{U_{0}^{*} V_{0}^{*}}{r^{*}}+2 \Omega_{D}^{*} U_{0}^{*}=\frac{1}{\rho^{*}} \frac{\partial}{\partial z^{*}}\left(\mu_{0}^{*} \frac{\partial V_{0}^{*}}{\partial z^{*}}\right), \\
\frac{\partial W_{0}^{*}}{\partial t^{*}}+U_{0}^{*} \frac{\partial W_{0}^{*}}{\partial r^{*}}+\frac{V_{0}^{*}}{r^{*}} \frac{\partial W_{0}^{*}}{\partial \theta}+W_{0}^{*} \frac{\partial W_{0}^{*}}{\partial z^{*}}=-\frac{1}{\rho^{*}} \frac{\partial P_{1}^{*}}{\partial z^{*}} \\
+\frac{1}{\rho^{*} r^{*}} \frac{\partial}{\partial r^{*}}\left(\mu_{0}^{*} r^{*} \frac{\partial U_{0}^{*}}{\partial z^{*}}\right)+\frac{1}{\rho^{*} r^{*}} \frac{\partial}{\partial \theta}\left(\mu_{0}^{*} \frac{\partial V_{0}^{*}}{\partial z^{*}}\right)+\frac{2}{\rho^{*}} \frac{\partial}{\partial z^{*}}\left(\mu_{0}^{*} \frac{\partial W_{0}^{*}}{\partial z^{*}}\right),
\end{gathered}
$$

Furthermore, the viscosity function $\mu_{0}^{*}$ is given by

$$
\mu_{0}^{*}=m^{*}\left[\left(\frac{\partial U_{0}^{*}}{\partial z^{*}}\right)^{2}+\left(\frac{\partial V_{0}^{*}}{\partial z^{*}}\right)^{2}\right]^{(n-1) / 2}
$$

and $\left(U_{0}^{*}, V_{0}^{*}, W_{0}^{*}, P_{1}^{*}\right)$ are the leading-order velocity and pressure components.

A generalisation of the exact von Kármán similarity solution is required in order to solve for the steady mean flow relative to the lower disk. This is expressed in non-dimensional form as

$$
\begin{aligned}
U(\eta) & =\frac{U_{0}^{*}}{r^{*} \triangle \Omega^{*}}=\frac{U_{0}^{*}}{r^{*} \Omega^{*} R o}, \\
V(\eta) & =\frac{V_{0}^{*}}{r^{*} \triangle \Omega^{*}}=\frac{V_{0}^{*}}{r^{*} \Omega^{*} R o}, \\
W(\eta) & =\frac{W_{0}^{*}}{\chi^{*}}, \quad P(\eta)=\frac{P_{1}^{*}}{\rho^{*}\left(\chi^{*}\right)^{2}},
\end{aligned}
$$

where

$$
\triangle \Omega^{*}=\Omega_{F}^{*}-\Omega_{D}^{*}, \quad \text { and } \quad \chi^{*}=\left[\frac{\nu^{*}}{\left(r^{*}\right)^{1-n}\left(\Omega^{*}\right)^{1-2 n} R o^{-2 n}}\right]^{1 /(n+1)} .
$$

Here $(U, V, W)$ are the dimensionless radial, azimuthal and axial velocities, respectively, $P$ is the pressure, and $\nu^{*}=m^{*} / \rho^{*}$ is the kinematic viscosity. The dimensionless similarity coordinate is defined by

$$
\eta=\frac{\left(r^{*}\right)^{(1-n) /(n+1)} z^{*}}{\left(L^{*}\right)^{2 /(n+1)}}, \quad \text { where } \quad L^{*}=\sqrt{\frac{\nu^{*}}{\left(\Omega^{*}\right)^{2-n} R o^{1-n}}},
$$

is the non-dimensional length scale.

The system rotation rate is given by

$$
\Omega^{*}=\frac{\Omega_{F}^{*}}{2-R o}+\frac{\Omega_{D}^{*}}{2+R o}=\frac{\Omega_{F}^{*}+\Omega_{D}^{*}}{4}+\left[\left(\frac{\Omega_{F}^{*}+\Omega_{D}^{*}}{4}\right)^{2}+\frac{\left(\triangle \Omega^{*}\right)^{2}}{2}\right]^{1 / 2} .
$$

These quantities define the Rossby number, Ro, which defines each particular flow within the BEK family

$$
R o=\frac{\triangle \Omega^{*}}{\Omega^{*}}=\frac{\Omega_{F}^{*}-\Omega_{D}^{*}}{\Omega^{*}}
$$


Defining also the Coriolis parameter, $C o=2 \Omega_{D}^{*} / \Omega^{*}=2-R o-R o^{2}$, leads to the particular flow cases being identified by

$\begin{array}{lll}\text { Bödewadt flow: } & R o=1 & C o=0 \\ \text { Ekman flow: } & R o=0 & C o=2 \\ \text { von Kármán flow: } & R o=-1 & C o=2\end{array}$

Substituting (4) into (3) gives the following non-dimensional equations for the mean flow:

$$
\begin{array}{r}
2 U+\frac{1-n}{n+1} \eta U^{\prime}+W^{\prime}=0, \\
R o\left[U^{2}-\left(V^{2}-1\right)+\left(W+\frac{1-n}{n+1} \eta U\right) U^{\prime}\right]-C o(V-1)-\left(\mu U^{\prime}\right)^{\prime}=0, \\
R o\left[2 U V+\left(W+\frac{1-n}{n+1} \eta U\right) V^{\prime}\right]+C o U-\left(\mu V^{\prime}\right)^{\prime}=0, \\
R o\left\{P^{\prime}+W W^{\prime}+\frac{1-n}{n+1}\left[U\left(\eta W^{\prime}-W\right)\right]\right\}+\frac{2(1-n)}{n+1} \mu U^{\prime}+2 \mu^{\prime} U-\left(\mu W^{\prime}\right)^{\prime}=0,
\end{array}
$$

where the primes denote derivatives with respect to $\eta$ and $\mu=\left[\left(U^{\prime}\right)^{2}+\left(V^{\prime}\right)^{2}\right]^{(n-1) / 2}$. When $n=1$ the system (8) is entirely consistent with the non-dimensional equations for the Newtonian mean flow given in the literature $[14,15,27]$.

The system of equations (8) are subject to the following boundary conditions

$$
\begin{array}{r}
U(\eta=0)=V(\eta=0)=W(\eta=0)=0, \\
U(\eta \rightarrow \infty)=V(\eta \rightarrow \infty)-1=0 .
\end{array}
$$

and the resulting non-linear ordinary differential equation system can be solved easily using a double-precision fourth-order Runge-Kutta integrator incorporating a Newton-Raphson searching method. Table 1 states the values of $U^{\prime}(0)$ and $V^{\prime}(0)$ arising from the shooting procedure. $^{1}$

The resulting mean-flow profiles are presented in Figure 1 for various values of $R o$. It is noted that the $(U, V, W)$ profiles for $R o=-1$ are identical to those obtained by Griffiths et al. $[22,23]$ which are not included here. Furthermore, when $n=1$, complete agreement is found at each $R o$ with the Newtonian profiles reported in the literature [14, 15, 27].

For $R o=-1$, only the radial mean flow is inflectional for all values of power-law index $n$. However, all mean flows (radial, azimuthal and axial) profiles become inflectional and the wall

\footnotetext{
${ }^{1}$ Note that where the BEK system exhibits non-uniqueness for particular Ro, we restrict our attention to the primary branch solution only.
} 
jet is increased with increasing $R o$ for all $n$. Furthermore, it is interesting to note that the oscillation of the mean flows is damped as the power-law index $n$ is reduced for all values of the Rossby number. 
Table 1: Numerical values of the mean velocity flow parameters $U^{\prime}(0), V^{\prime}(0)$ and $W\left(\eta_{\infty}\right)$ for $n=$ $1,0.9,0.8,0.7,0.6$ and for various $R o$. The value of $\eta_{\infty}$ is chosen such that full converged mean flow solutions are obtained.

\begin{tabular}{|c|c|c|c|c|}
\hline & $n$ & $-U^{\prime}(0)$ & $V^{\prime}(0)$ & $W\left(\eta_{\infty}\right)$ \\
\hline$R o=-1$ & 1.0 & 0.5102 & 0.6159 & 0.8845 \\
\hline \multirow[t]{4}{*}{ von Kármán flow } & 0.9 & 0.5069 & 0.6243 & 0.9698 \\
\hline & 0.8 & 0.5039 & 0.6362 & 1.0957 \\
\hline & 0.7 & 0.5017 & 0.6532 & 1.3051 \\
\hline & 0.6 & 0.5005 & 0.6778 & 1.7329 \\
\hline \multirow[t]{5}{*}{$R o=-0.5$} & 1.0 & 0.8570 & 0.9073 & 1.0219 \\
\hline & 0.9 & 0.8687 & 0.9434 & 1.0241 \\
\hline & 0.8 & 0.8831 & 0.9885 & 1.0250 \\
\hline & 0.7 & 0.9013 & 1.0461 & 1.0241 \\
\hline & 0.6 & 0.9245 & 1.1221 & 1.0205 \\
\hline$R o=0$ & 1.0 & 1.0000 & 1.0000 & 1.0000 \\
\hline \multirow[t]{4}{*}{ Ekman flow } & 0.9 & 1.0189 & 1.0469 & 0.9814 \\
\hline & 0.8 & 1.0418 & 1.1050 & 0.9599 \\
\hline & 0.7 & 1.0697 & 1.1787 & 0.9348 \\
\hline & 0.6 & 1.1046 & 1.2755 & 0.9053 \\
\hline \multirow[t]{5}{*}{$R o=0.5$} & 1.0 & 1.0176 & 0.9612 & 1.0853 \\
\hline & 0.9 & 1.0346 & 1.0057 & 1.0567 \\
\hline & 0.8 & 1.0550 & 1.0607 & 1.0246 \\
\hline & 0.7 & 1.0798 & 1.1304 & 0.9882 \\
\hline & 0.6 & 1.1108 & 1.2212 & 0.9468 \\
\hline$R o=1$ & 1.0 & 0.9420 & 0.7729 & 1.3494 \\
\hline \multirow[t]{4}{*}{ Bödewadt flow } & 0.9 & 0.9442 & 0.8021 & 1.3201 \\
\hline & 0.8 & 0.9475 & 0.8376 & 1.2855 \\
\hline & 0.7 & 0.9526 & 0.8821 & 1.2446 \\
\hline & 0.6 & 0.9599 & 0.9398 & 1.1962 \\
\hline
\end{tabular}



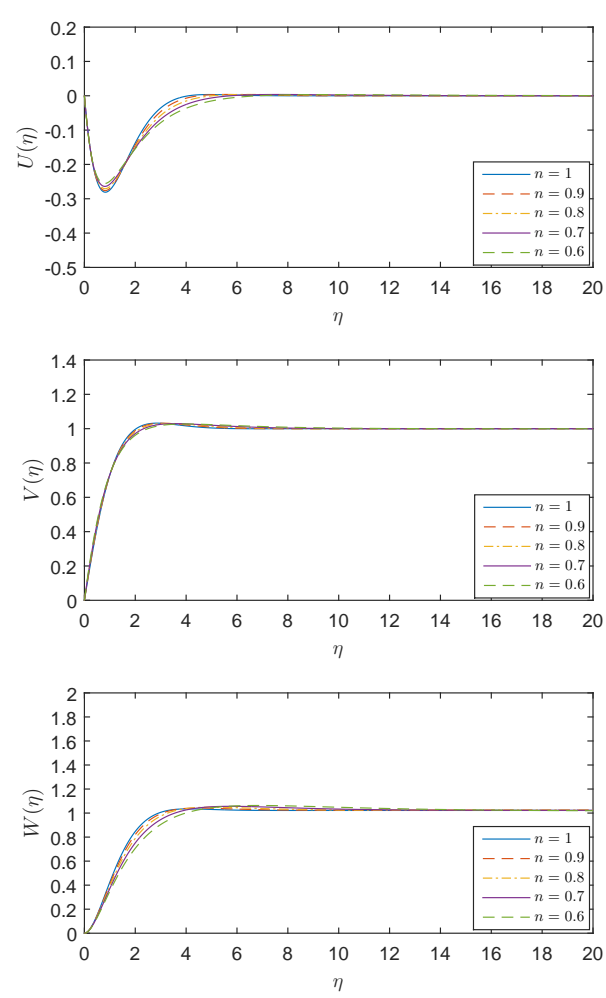

(a) $R o=-0.5$
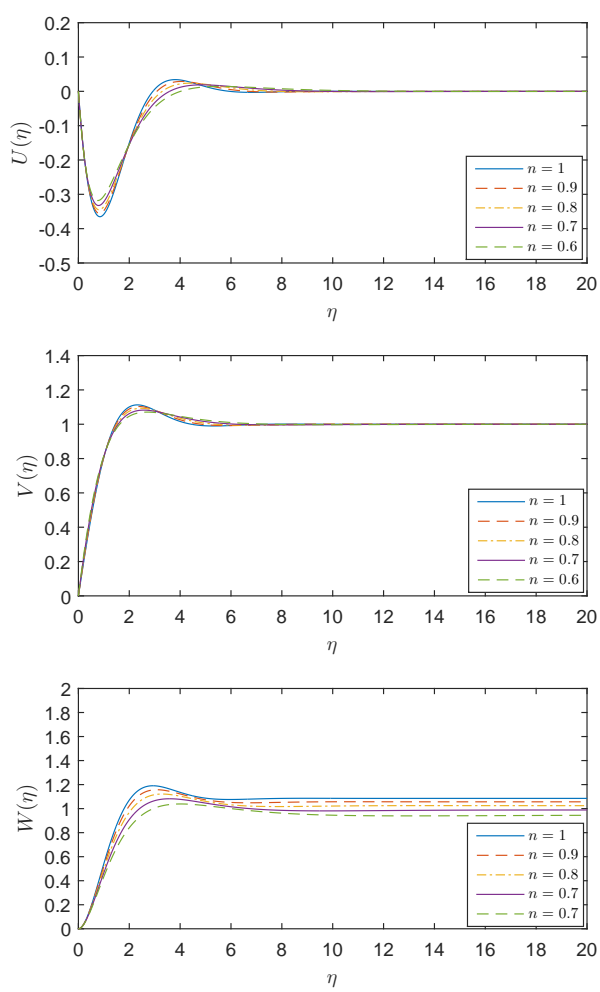

(c) $R o=0.5$
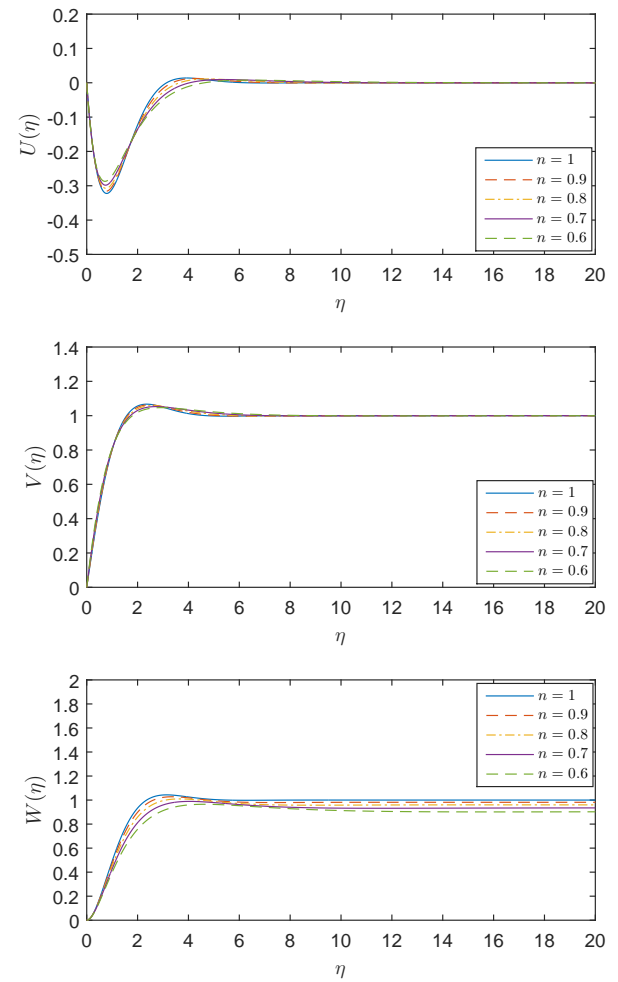

(b) Ekman, Ro $=0$
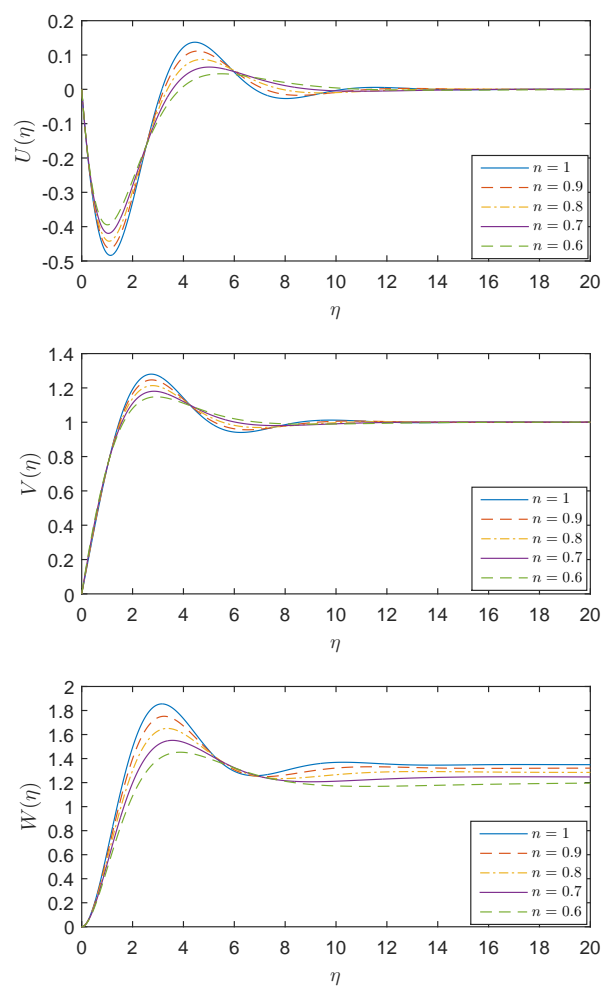

(d) Bödewadt, $R o=1$

Figure 1: Plots of $U, V$ and $W$ versus $\eta$ for Newtonian and shear-thinning power-law fluids with $n=1,0.9,0.8,0.7,0.6$. 


\section{Convective instability analysis}

A local linear stability analysis is applied at a radius $r_{a}^{*}$ by imposing infinitesimally small disturbances on the steady-mean flow at that position. Here the local Reynolds number is defined as

$$
\begin{aligned}
R & =\left(r_{a}^{*}\right)^{2 /(n+1)}\left[\frac{\left(\triangle \Omega^{*}\right)^{2-n} L^{*}}{\nu^{*}}\right]^{2 /(n+1)}=\left(r_{a}^{*}\right)^{2 /(n+1)}\left[\frac{R o^{2-n}\left(\Omega^{*}\right)^{2-n} L^{*}}{\nu^{*}}\right]^{2 /(n+1)} \\
& =\left(r_{a}^{*}\right)^{2 /(n+1)}\left[\frac{R o R o^{1-n}\left(\Omega^{*}\right)^{2-n} L^{*}}{\nu^{*}}\right]^{2 /(n+1)}=\left(\frac{r_{a}^{*} R o}{L^{*}}\right)^{2 /(n+1)} \\
& =\left(r_{a} R o\right)^{2 /(n+1)}
\end{aligned}
$$

Note that this definition is such that the Reynolds number is negative for Newtonian flow $(n=1)$ when the Rossby number is negative. However, this is simply a consequence of the formulation and all results will be given in terms of positive $R$ for all $R o$ and $n$. We see that the Reynolds number can be interpreted as the non-dimensional location of the local analysis, and increasing $R$ in magnitude corresponds to moving radially outwards from the axis of rotation (irrespective of the sign of the Rossby number).

The non-dimensionalising velocity, pressure and time scales are $r_{a}^{*} \Omega^{*} R o, \rho^{*}\left(r_{a}^{*} \Omega^{*} R o\right)^{2}$ and $L^{*} /\left(r_{a}^{*} \Omega^{*} R o\right)$, respectively. The leading-order pressure terms in the radial and azimuthal momentum equations are retained, allowing for the inclusion of the disturbance pressure terms in the respective linear disturbance equations. The instantaneous non-dimensional velocities and pressure are defined as

$$
\begin{aligned}
U_{0}(\eta, r, \theta, t) & =\frac{r R o}{R^{(n+1) / 2}} U(\eta)+u(\eta, r, \theta, t), \\
V_{0}(\eta, r, \theta, t) & =\frac{r R o}{R^{(n+1) / 2}} V(\eta)+v(\eta, r, \theta, t), \\
W_{0}(\eta, r, \theta, t) & =\frac{r^{(n-1) /(n+1)} R o}{R^{(n+1) / 2}} W(\eta)+w(\eta, r, \theta, t), \\
P_{1}(\eta, r, \theta, t) & =\frac{r^{2(n-1) /(n+1)} R^{2}}{R^{(n+1)}} P(\eta)+p(\eta, r, \theta, t),
\end{aligned}
$$

where $\eta=\eta(r, z)=r^{(1-n) /(n+1)} z$ and $u, v, w$ and $p$ are small perturbing quantities.

Note that the scalings used in (4), (5) and (10) are a generalisation of the similarity solution introduced by Mitschka and Ulbrecht [19] for the von Kármán system under the power-law model. When $R_{0}=-1$, these expressions are consistent with those used by Griffiths et al. $[22,23]$, and, when $n=1$, they are consistent with those used in the Newtonian study of the BEK system by Lingwood [14].

The dimensionless Navier-Stokes equations in cylindrical-polar coordinates are linearised with respect to the perturbation quantities. The parallel-flow approximation is applied to 
ensure that the linearised equations are separable in $r, \theta$ and $t$. In practice this involves ignoring variations in the Reynolds number with radius by replacing the variable $r$ with $R^{(n+1) / 2} / R_{o}$, and by neglecting the $\left.\mathcal{O}\left(\left(R_{o} / R\right)\right)^{2}\right)$ terms. Furthermore, it has been necessary to set each factor $R o^{j}$ to unity, where $j$ is some expression involving $n-1$. This approximation is necessary to insure the continuity as $R o$ is varied from -1 to 1 . The resulting linear disturbance equations are given by

$$
\begin{gathered}
R^{(n-1) / 2} \frac{\partial u}{\partial r}+\frac{R o}{R}\left[\eta \frac{(1-n)}{(n+1)} \frac{\partial u}{\partial \eta}+u+\frac{\partial v}{\partial \theta}\right]+\frac{\partial w}{\partial \eta}=0, \\
R^{(n-1) / 2}\left(\frac{\partial u}{\partial t}+U \frac{\partial u}{\partial r}\right)+\frac{R o}{R}\left(V \frac{\partial u}{\partial \theta}+W \frac{\partial u}{\partial \eta}+U u\right)+U^{\prime} w-\frac{(2 R o V+C o) v}{R} \\
+\frac{R o}{R} \eta \frac{(1-n)}{(n+1)}\left(U \frac{\partial u}{\partial \eta}+U^{\prime} u+\frac{\partial p}{\partial \eta}\right)=-R^{(n-1) / 2} \frac{\partial p}{\partial r}+\frac{1}{R} \frac{\partial}{\partial \eta}\left(\mu \frac{\partial u}{\partial \eta}+\hat{\mu} U^{\prime}\right), \\
R^{(n-1) / 2}\left(\frac{\partial v}{\partial t}+U \frac{\partial v}{\partial r}\right)+\frac{R o}{R}\left(V \frac{\partial v}{\partial \theta}+W \frac{\partial v}{\partial \eta}+U v\right)+V^{\prime} w+\frac{(2 R o V+C o) u}{R} \\
+\frac{R o}{R} \eta \frac{(1-n)}{(n+1)}\left(U \frac{\partial v}{\partial \eta}+V^{\prime} u\right)=-\frac{R o}{R} \frac{\partial p}{\partial \theta}+\frac{1}{R} \frac{\partial}{\partial \eta}\left(\mu \frac{\partial v}{\partial \eta}+\hat{\mu} V^{\prime}\right), \\
R^{(n-1) / 2}\left(\frac{\partial w}{\partial t}+U \frac{\partial w}{\partial r}\right)+\frac{R o}{R}\left[V \frac{\partial w}{\partial \theta}+W \frac{\partial w}{\partial \eta}+W^{\prime} w+\eta \frac{(1-n)}{(n+1)} U \frac{\partial w}{\partial \eta}\right] \\
=-\frac{\partial p}{\partial \eta}+\frac{1}{R} \frac{\partial}{\partial \eta}\left(\mu \frac{\partial w}{\partial \eta}\right)=0,
\end{gathered}
$$

where the disturbance viscosity function is given by

$$
\hat{\mu}=\frac{(n-1) \mu}{\left(U^{\prime}\right)^{2}+\left(V^{\prime}\right)^{2}}\left(U^{\prime} \frac{\partial u}{\partial \eta}+V^{\prime} \frac{\partial v}{\partial \eta}\right) .
$$

We now suppose that the perturbation quantities have the normal-mode form consistent with the prior non-Newtonian analyses [14, 15, 27],

$$
u=\hat{u}(\eta ; \alpha, \beta, \omega ; R, R o) e^{\mathrm{i}\left(\alpha r+\beta^{*} \theta-\omega t\right)},
$$

and similarly for $v, w$ and $p$. Here $\hat{u}, \hat{v}, \hat{w}$ and $\hat{p}$ are the spectral representations of the perturbation velocities and pressure, respectively, $\alpha=\alpha_{\mathrm{r}}+\mathrm{i} \alpha_{\mathrm{i}}$ and $\beta^{*}=\beta / R o$ are the complex radial and real azimuthal wave-numbers, and $\omega$ is the frequency of the disturbance in the frame rotating with the lower disk. The perturbation equations now reduce to

$$
\left(\mathrm{i} \bar{\alpha}+\frac{R o}{R}\right) \hat{u}+\mathrm{i} \bar{\beta} \hat{v}+\frac{R o}{R} \eta \frac{(1-n)}{(n+1)} \frac{\partial \hat{u}}{\partial \eta}+\frac{\partial \hat{w}}{\partial \hat{\eta}}=0,
$$




$$
\begin{gathered}
{\left[\mathrm{i}(\bar{\alpha} U+\bar{\beta} V-\bar{\omega})+\frac{R o}{R} U\right] \hat{u}-\frac{(2 R o V+C o) \hat{v}}{R}+\frac{R o}{R} W \frac{\partial \hat{u}}{\partial \eta}+U^{\prime} \hat{w}} \\
+\frac{R o}{R} \eta \frac{(1-n)}{(n+1)}\left(U \frac{\partial \hat{u}}{\partial \eta}+U^{\prime} \hat{u}+\frac{\partial p}{\partial \eta}\right)+\mathrm{i} \bar{\alpha} \hat{p}-\frac{1}{R} \frac{\partial}{\partial \eta}\left(\mu \frac{\partial \hat{u}}{\partial \eta}+\hat{\mu} U^{\prime}\right)=0 \\
{\left[\mathrm{i}(\bar{\alpha} U+\bar{\beta} V-\bar{\omega})+\frac{R o}{R} U\right] \hat{v}+\frac{(2 R o V+C o) \hat{u}}{R}+\frac{R o}{R} W \frac{\partial \hat{v}}{\partial \eta}+V^{\prime} \hat{w}} \\
+\frac{R o}{R} \eta \frac{(1-n)}{(n+1)}\left(U \frac{\partial \hat{v}}{\partial \eta}+V^{\prime} \hat{u}\right)+i \bar{\beta} \hat{p}-\frac{1}{R} \frac{\partial}{\partial \eta}\left(\mu \frac{\partial \hat{v}}{\partial \eta}+\hat{\mu} V^{\prime}\right)=0 \\
{\left[\mathrm{i}(\bar{\alpha} U+\bar{\beta} V-\bar{\omega})+\frac{R o}{R} W^{\prime}\right] \hat{w}+\frac{R o}{R}\left[W \frac{\partial \hat{w}}{\partial \eta}+\eta \frac{(1-n)}{(n+1)} U \frac{\partial \hat{w}}{\partial \eta}\right]} \\
+\frac{\partial \hat{p}}{\partial \eta}-\frac{1}{R} \frac{\partial}{\partial \eta}\left(\mu \frac{\partial \hat{w}}{\partial \eta}\right)=0
\end{gathered}
$$

where $\bar{\alpha}=R^{(n-1) / 2} \alpha, \bar{\beta}=\beta / R$ and $\bar{\omega}=R^{(n-1) / 2} \omega$.

The modified wavenumber and orientation angle typically used in asymptotic analyses (see Griffiths et al. [22]) are then given by

$$
\kappa=\sqrt{\bar{\alpha}^{2}+\bar{\beta}^{2}}=r^{(n-1) /(n+1)} \sqrt{\alpha^{2}+\frac{\beta^{2}}{r^{2}}},
$$

and

$$
\phi=\tan ^{-1}\left(\frac{\bar{\beta}}{\bar{\alpha}}\right) \Leftrightarrow \tan \left(\frac{\pi}{2}-\phi\right)=\frac{\alpha r}{\beta} .
$$

Note that additional viscous terms $\hat{\mu} U^{\prime}$ and $\hat{\mu} V^{\prime}$ appear in our perturbation equations (13) and these are due to the first-order terms of the cross-product associated with the generalised binomial expansion of the perturbed viscosity function. The study of Griffiths et al. [23] neglects these terms along with other $\eta / R$ terms. However, here our solution method is such that all $\hat{\mu} U^{\prime}, \hat{\mu} V^{\prime}$ and $\eta / R$ terms can be retained. This represents a small deviation from Griffiths' prior study of the von Kármán flow $(R o=-1)$ but should be considered as an improvement. Consistent with Griffiths' analyses, we note that when $n=1$, the perturbation equations are not entirely consistent with the Newtonian set of transformed perturbation equations used by Lingwood [14], Lingwood and Garrett [15] for Newtonian flows. This minor discrepancy is due to the boundary-layer approximation used to construct the steady mean-flow solutions in this general non-Newtonian formulation. Further details on this are given in the literature [23].

In much the same way as presented by Alveroglu et al. [27], we solve the perturbation equations (13) using a Galerkin projection in terms of Chebyshev polynomials. This enables solutions of the dispersion relation $D(\alpha, \beta, \omega ; R, n, R o)=0$ to be obtained at each $(R, n, R o)$ with the aim of studying the occurrence of local convective instability at each parameter triple. 
Chebyshev polynomials permit significantly higher accuracy than standard finite-difference methods and this motivates their use here. Furthermore, as discussed by Alveroglu et al. [27], this approach has two additional advantages over the shooting method used in the literature $[14,15,23]$. Firstly, the perturbation equations are solved in terms of primitive variables not transformed to a new system; this permits the retention of a number of terms otherwise neglected (as discussed previously). Secondly, all eigenvalues can be obtained simultaneously instead of searching iteratively from some initial guess.

Consistent with all other stability analyses in the literature, the perturbation equations (13) are subject to boundary conditions

$$
\begin{array}{r}
\hat{u}(\eta=0)=\hat{v}(\eta=0)=\hat{w}(\eta=0)=0 \\
\hat{u}\left(\eta \rightarrow \eta_{\infty}\right)=\hat{v}\left(\eta \rightarrow \eta_{\infty}\right)=\hat{w}\left(\eta \rightarrow \eta_{\infty}\right)=0
\end{array}
$$

These represent the no-slip condition on disk surface and ensure that the disturbances are contained within the boundary layer. All calculations use a Gauss-Lobatto grid with 100 points distributed via an exponential map between the lower disk surface $\eta=0$ and the top of the domain $\eta=\eta_{\infty}$. Further increases in the resolution and spatial extent of this grid were found to have no numerical effect on the stability results.

\subsection{Neutral curves and critical Reynolds numbers}

Neutral curves, defined by the neutral spatial growth $\alpha_{\mathrm{i}}=0$, have been calculated for $0.6 \leq n \leq 1$ in increments of 0.1 at $R o=-1,-0.5,0,0.5$ and 1 . This range is deemed sufficient to capture the convective instability characteristics of shear-thinning flows within the BEK family. Figure 2 present the neutral curves in the $R-\kappa$ and $R-\phi$ planes where $\kappa$ and $\phi$ denote the wavenumber and wave angle, respectively, and are defined in (14) and (15). The neutral curves are seen to have a two-lobed structure demonstrating that both the type I and type II modes exist in the non-Newtonian system. In particular, the upper lobes (in the $R$-wavenumber planes) correspond to the type I mode and the smaller lower lobes to the type II mode. Critical Reynolds numbers for the onset of both modes are presented in Table 2. We note that Figure 2(a) is consistent with the neutral curves generated by Griffiths et al. [23] for Newtonian disk flow, $R o=-1$ and $n=1$. However, slight differences in the numerical values of the critical Reynolds numbers are found owing to our inclusion of the additional viscous terms $\hat{\mu} U^{\prime}, \hat{\mu} V^{\prime}$ and $\eta / R$ in the perturbation equations. It is interesting to note that these terms lead to growth in the relative size of the type II mode as $n$ is decreased (as compared to Griffiths' results). In addition, the critical Reynolds numbers are reduced in comparison with Griffiths' numerical results (as predicted by [22]). 
The neutral curves in Figure 2 and critical Reynolds numbers in Table 2 suggest that decreasing the power-law index $(n<1)$ has a stabilising effect on both the dominant type I mode and secondary type II mode for all Ro. That is, shear-thinning fluids act to stabilise the von Kármán, the Ekman, the Bödewadt, and all intermediate boundary layers. Figure 2(b), (c), (d) and (e) further suggests that, while reducing the associated critical Reynolds number, reductions in $n$ act to promote the appearance of the distinct type II mode. 
Table 2: The values of the critical Reynolds number $R$, wavenumbers $\bar{\alpha}, \bar{\beta}$ and $\kappa$ and wave angle $\phi$ corresponding to decreasing values of $n$ on the both modes type I and (type II).

\begin{tabular}{|c|c|c|c|c|c|c|}
\hline & $n$ & $R$ & $\bar{\alpha}$ & $\bar{\beta}$ & $\kappa$ & $\phi$ \\
\hline$R o=-1$ & 1.0 & $272.90(445.21)$ & $0.4029(0.1319)$ & $0.0802(0.0467)$ & $0.4108(0.1399)$ & $11.26(19.48)$ \\
\hline \multirow[t]{4}{*}{ von Kármán flow } & 0.9 & $323.60(566.41)$ & $0.3768(0.1142)$ & $0.0781(0.0419)$ & $0.3848(0.1216)$ & $11.71(20.16)$ \\
\hline & 0.8 & $392.36(741.16)$ & $0.3518(0.0982)$ & $0.0760(0.0374)$ & $0.3599(0.1051)$ & $12.19(20.83)$ \\
\hline & 0.7 & $488.20(999.78)$ & $0.3298(0.0841)$ & $0.0743(0.0331)$ & $0.3380(0.0903)$ & $12.69(21.46)$ \\
\hline & 0.6 & $626.06(1394.57)$ & $0.3103(0.0717)$ & $0.0725(0.0290)$ & $0.3186(0.0773)$ & $13.15(22.01)$ \\
\hline \multirow[t]{5}{*}{$R o=-0.5$} & 1.0 & $149.74(-)$ & $0.5098(-)$ & $0.1237(-)$ & $0.5246(-)$ & $13.64(-)$ \\
\hline & 0.9 & $180.37(-)$ & $0.4780(-)$ & $0.1185(-)$ & $0.4924(-)$ & $13.92(-)$ \\
\hline & 0.8 & $221.84(-)$ & $0.4495(-)$ & $0.1139(-)$ & $0.4637(-)$ & $14.22(-)$ \\
\hline & 0.7 & $279.54(-)$ & $0.4253(-)$ & $0.1102(-)$ & $0.4393(-)$ & $14.52(-)$ \\
\hline & 0.6 & $362.69(763.26)$ & $0.4065(0.1179)$ & $0.1075(0.0527)$ & $0.4204(0.1291)$ & $15.81(24.08)$ \\
\hline$R o=0$ & 1.0 & $105.88(-)$ & $0.5805(-)$ & $0.1444(-)$ & $0.5982(-)$ & $13.97(-)$ \\
\hline \multirow[t]{4}{*}{ Ekman flow } & 0.9 & $127.54(-)$ & $0.5466(-)$ & $0.1386(-)$ & $0.5639(-)$ & $14.23(-)$ \\
\hline & 0.8 & $157.52(-)$ & $0.5163(-)$ & $0.1336(-)$ & $0.5333(-)$ & $14.51(-)$ \\
\hline & 0.7 & $200.20(-)$ & $0.4891(-)$ & $0.1293(-)$ & $0.5059(-)$ & $14.81(-)$ \\
\hline & 0.6 & $263.00(496.81)$ & $0.4677(0.1569)$ & $0.1263(0.0704)$ & $0.4845(0.1720)$ & $15.11(24.15)$ \\
\hline \multirow[t]{5}{*}{$R o=0.5$} & 1.0 & $67.21(-)$ & $0.5861(-)$ & $0.1455(-)$ & $0.6039(-)$ & $13.94(-)$ \\
\hline & 0.9 & $81.28(-)$ & $0.5521(-)$ & $0.1402(-)$ & $0.5696(-)$ & $14.25(-)$ \\
\hline & 0.8 & $101.40(-)$ & $0.5187(-)$ & $0.1351(-)$ & $0.5360(-)$ & $14.60(-)$ \\
\hline & 0.7 & $131.08(-)$ & $0.4892(-)$ & $0.1306(-)$ & $0.5063(-)$ & $14.95(-)$ \\
\hline & 0.6 & $176.36(-)$ & $0.4646(-)$ & $0.1271(-)$ & $0.4816(-)$ & $15.30(-)$ \\
\hline$R o=1$ & 1.0 & $22.40(-)$ & $0.5404(-)$ & $0.1185(-)$ & $0.5532(-)$ & $12.37(-)$ \\
\hline \multirow[t]{4}{*}{ Bödewadt flow } & 0.9 & $27.22(-)$ & $0.5119(-)$ & $0.1189(-)$ & $0.5256(-)$ & $13.07(-)$ \\
\hline & 0.8 & $34.51(-)$ & $0.4803(-)$ & $0.1179(-)$ & $0.4946(-)$ & $13.79(-)$ \\
\hline & 0.7 & $46.08(-)$ & $0.4470(-)$ & $0.1155(-)$ & $0.4616(-)$ & $14.49(-)$ \\
\hline & 0.6 & $65.71(-)$ & $0.4108(-)$ & $0.1117(-)$ & $0.4257(-)$ & $15.21(-)$ \\
\hline
\end{tabular}



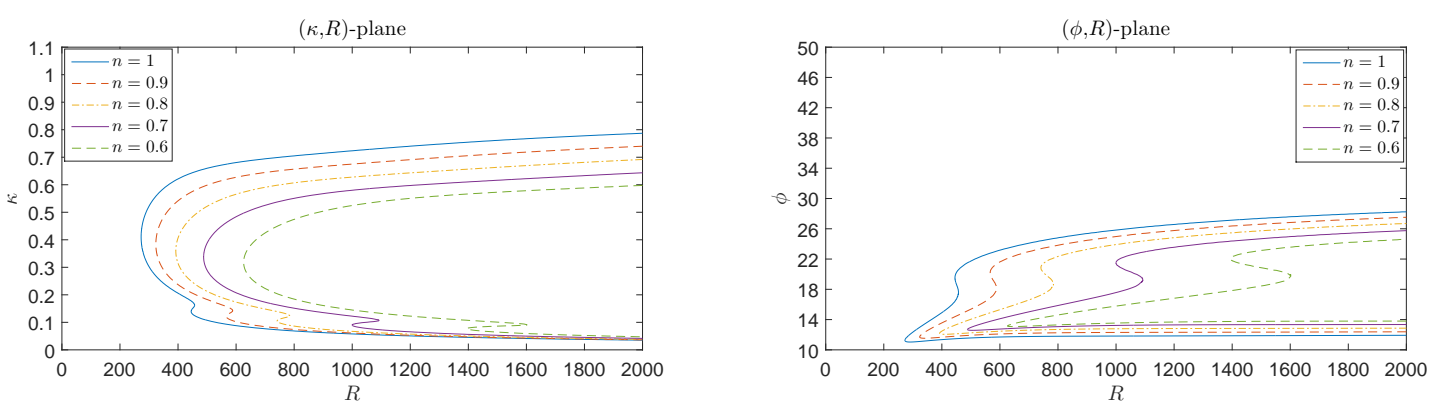

(a) von Kármán, $R o=-1$
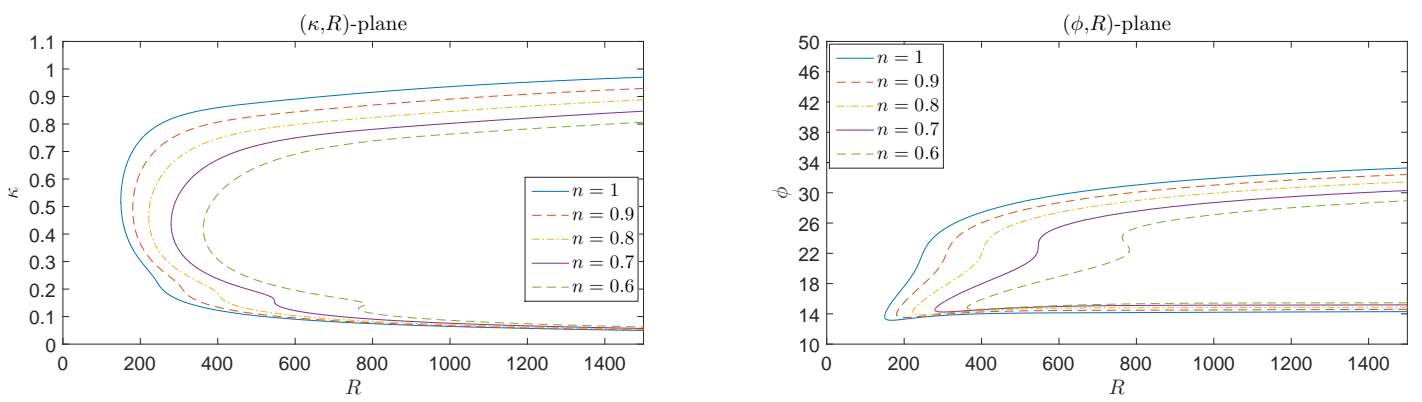

(b) $R o=-0.5$
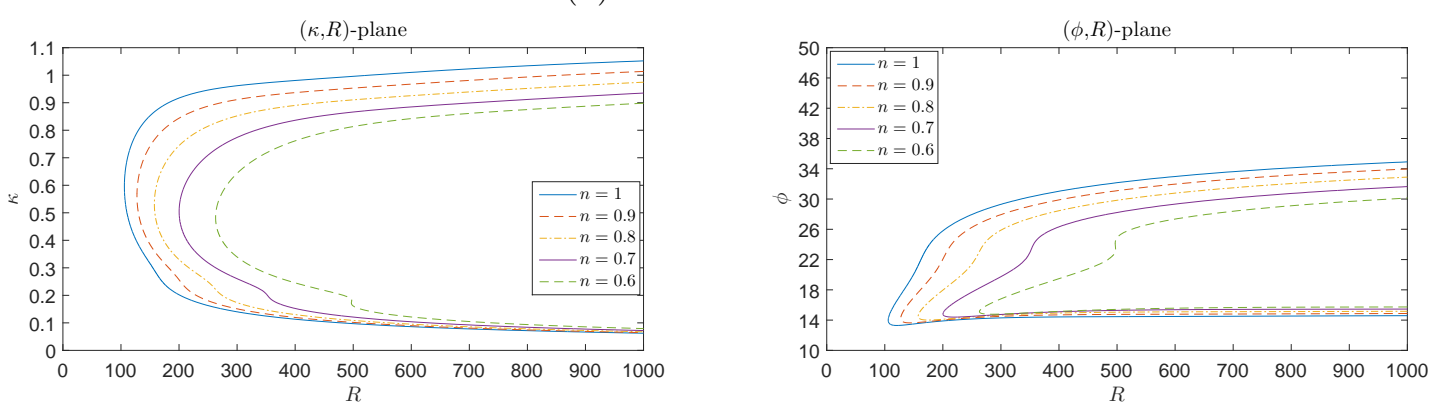

(c) Ekman, $R o=0$
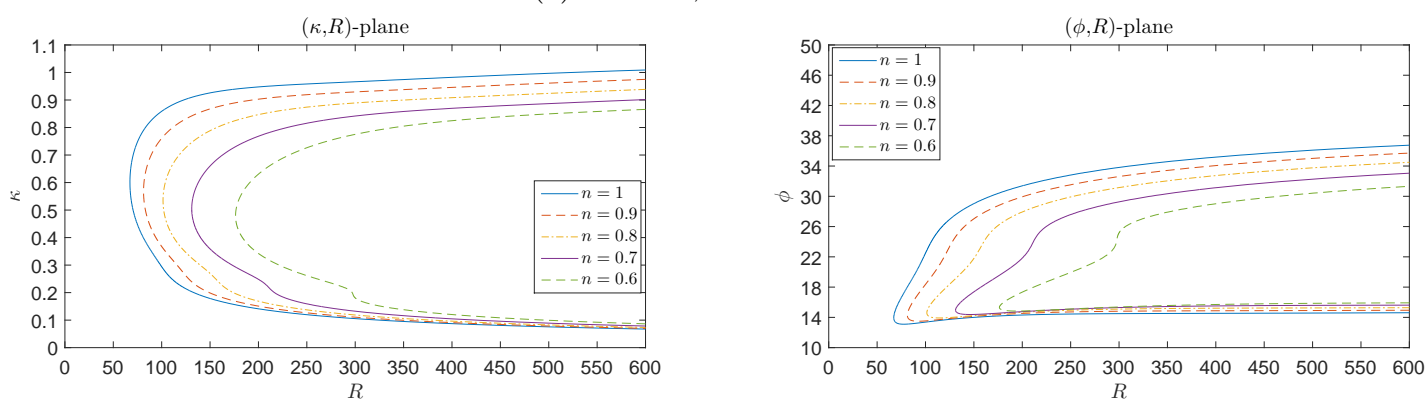

(d) $R o=0.5$
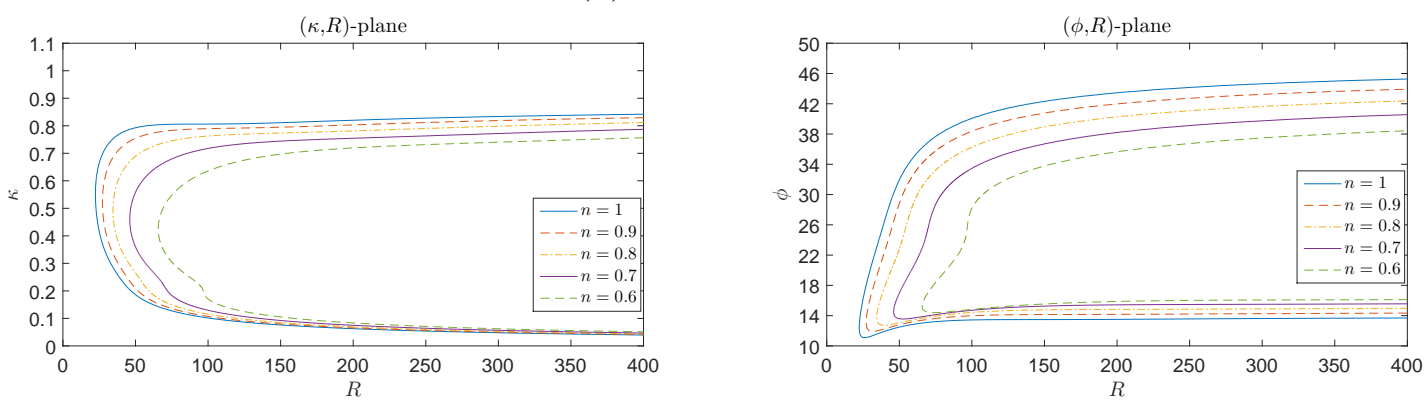

(e) Bödewadt, $R o=1$

Figure 2: Neutral curves for Newtonian and shear-thinning power-law fluids with $n=1,0.9,0.8,0.7,0.6$. 


\subsection{Convective growth rates}

Figure 3 presents the convective growth rates for the dominant type I mode at $R=R_{c}+25$ against $\bar{n}$ for $R o=-1,-0.5,0$ and for variousvalues of $n$. Here $\bar{n}=\bar{\beta} R$ is the number of spiral vortices around the disk surface, and $R_{c}$ denotes the critical Reynolds number for the onset of the type I mode for the particular $n$ and $R o$. That is, we sample the growth rate a fixed distance into the convectively unstable region for a variety of flow configurations. The figure reveals a universal stabilising effect on the growth rates as $n$ decreases; this is in addition to the stabilising increase in $R_{c}$ with reduced $n$ as identified previously.

It is interesting to note that the maximum growth rate (identified with a red dot) is pushed to a higher $\bar{n}$. That is, although we might expect the delayed onset and weaker growth of the disturbances for shear-thinning fluids, the number of spiral vortices, at the maximal growth rate location is comparatively larger. We return to these most amplified modes in the energy analysis of Section 4.

Note that it is not possible to show convective growth rates for $R o \geq 0.5$. This is due to the very early onset of absolute instability and associated "branch exchange" at these positive values of $R o$. Further information on this can be found in the corresponding Newtonian study performed by Lingwood and Garrett [15]. 


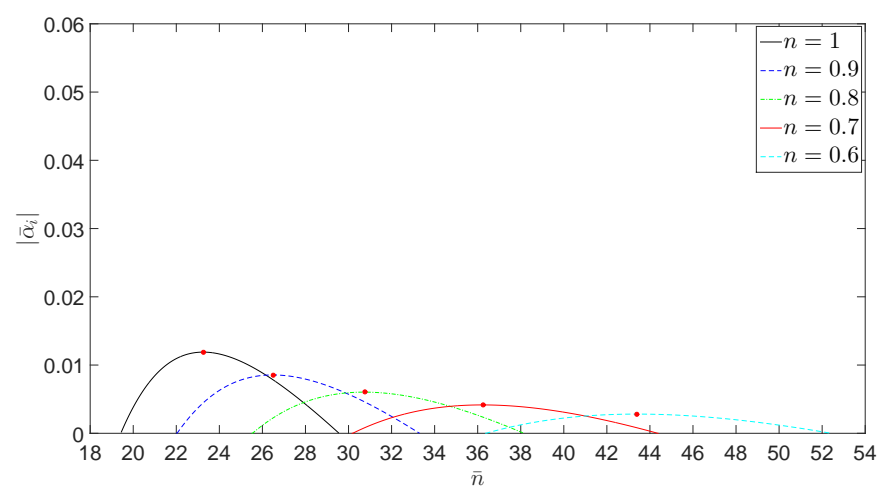

(a) von Kármán, $R o=-1$

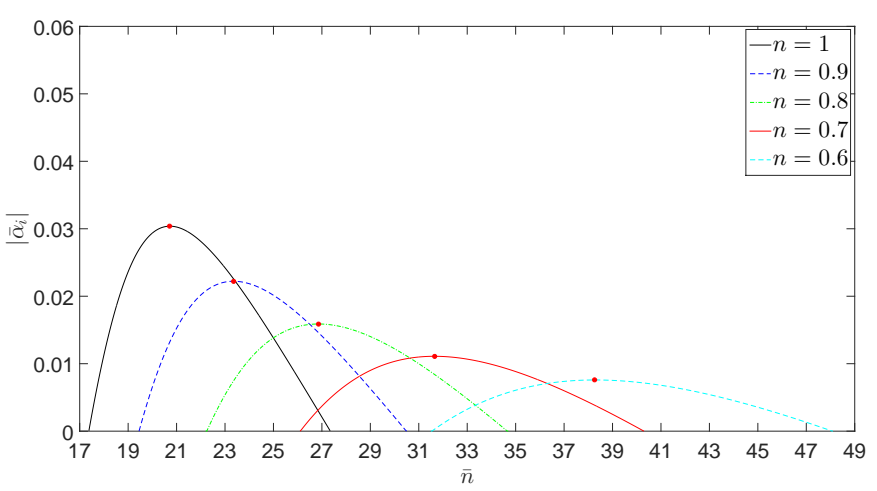

(b) $R o=-0.5$

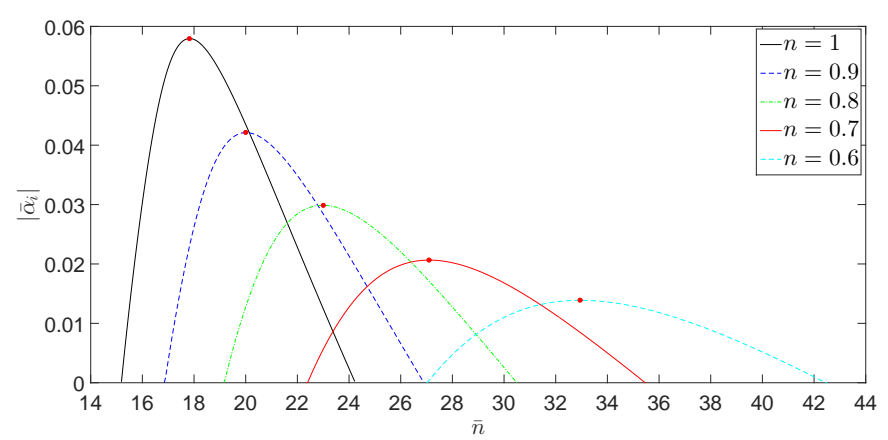

(c) Ekman, $R o=0$

Figure 3: Growth rates for type I mode for power-law fluids with $n=1,0.9,0.8,0.7,0.6$. 


\section{Energy analysis}

Following the work of Cooper and Carpenter [28], Cooper et al. [29] and Garrett et al. [30], an integral energy equation for the disturbances within the power-law BEK family is derived in order to gain insight into the underlying physical mechanisms behind the stabilising effects determined previously.

We follow Cooper's approach and derive the governing energy equations by multiplying the linearised momentum equations (11) by the disturbance quantities $u, v$ and $w$, respectively. These are then summed to obtain the following kinetic-energy equation for the disturbances

$$
\begin{aligned}
& {\left[R^{(n-1) / 2}\left(\frac{\partial}{\partial t}+U \frac{\partial}{\partial r}\right)+\frac{R o}{R}\left(V \frac{\partial}{\partial \theta}-W \frac{\partial}{\partial \eta}\right)\right] K=-w\left(u U^{\prime}+v V^{\prime}\right)} \\
& +\frac{R o}{R}\left[W^{\prime} w^{2}+U\left(u^{2}+v^{2}\right)\right]-\left\{R^{(n-1) / 2} \frac{\partial(u p)}{\partial r}+\frac{R o}{R}\left[\frac{\partial(v p)}{\partial \theta}-u p\right]+\frac{\partial(w p)}{\partial \eta}\right\} \\
& +\left(\frac{\partial u_{j} \sigma_{i j}}{\partial x_{i}}-\sigma_{i j} \frac{\partial u_{j}}{\partial x_{i}}\right)+\frac{1}{R}\left[\frac{\partial}{\partial \eta}\left(\mu \frac{\partial u}{\partial \eta}\right) u+\frac{\partial}{\partial \eta}\left(\mu \frac{\partial v}{\partial \eta}\right) v+\frac{\partial}{\partial \eta}\left(\mu \frac{\partial w}{\partial \eta}\right) w\right] .
\end{aligned}
$$

Here $K=(1 / 2)\left(u^{2}+v^{2}+w^{2}\right)$ is the kinetic energy and $\sigma_{i j}$ are the anti-symmetric viscous stress terms

$$
\sigma_{i j}=\frac{1}{R}\left(\frac{\partial u_{i}}{\partial x_{j}}-\frac{\partial u_{j}}{\partial x_{i}}\right)
$$

Note that the $\mathcal{O}\left(1 / R^{2}\right)$ viscous terms have been omitted to ensure consistency with the linearised governing stability equations. Furthermore, the derivatives with respect to $t$ and $\theta$ are removed to obtain the steady, rotationally-symmetric nature of the disturbances. Moreover, we find that terms involving the function $\hat{\mu}$ can be neglected because of their negligible effect on the stability of the flow. The perturbations are averaged over a single time period and azimuthal mode and are then integrated across the entire boundary layer to obtain the following expression 


$$
\begin{aligned}
& \int_{0}^{\infty}[\underbrace{R^{(n-1) / 2} U \frac{\partial \bar{K}}{\partial r}}_{a}+\underbrace{R^{(n-1) / 2} \frac{\partial(\overline{u p})}{\partial r}}_{b}-\underbrace{\frac{\partial}{\partial r}\left(\overline{u \sigma_{11}}+\overline{v \sigma_{12}}+\overline{w \sigma_{13}}\right)}_{c}] \mathrm{d} \eta \\
& =\underbrace{\int_{0}^{\infty}\left(\frac{R o}{R} \overline{w^{2}} \frac{\partial W}{\partial \eta}-\overline{u w} \frac{\partial U}{\partial \eta}-\overline{v w} \frac{\partial V}{\partial \eta}\right) \mathrm{d} \eta}_{\text {I }}-\underbrace{\int_{0}^{\infty} \overline{\sigma_{i j} \frac{\partial u_{j}}{\partial x_{i}}} \mathrm{~d} \eta}_{\text {II }} \\
& +\underbrace{\frac{R o}{R} \int_{0}^{\infty} \overline{u p} \mathrm{~d} \eta+(\overline{w p})_{W}}_{\text {III }}-\underbrace{\left(\overline{u \sigma_{31}}+\overline{v \sigma_{32}}+\overline{w \sigma_{33}}\right)_{W}}_{\text {IV }} \\
& +\underbrace{\frac{R o}{R}\left[\int_{0}^{\infty} \overline{u^{2}} U \mathrm{~d} \eta+\int_{0}^{\infty} \overline{v^{2}} U \mathrm{~d} \eta+\int_{0}^{\infty} \frac{\partial \bar{K}}{\partial \eta} W \mathrm{~d} \eta\right]}_{\mathrm{V}}
\end{aligned}
$$

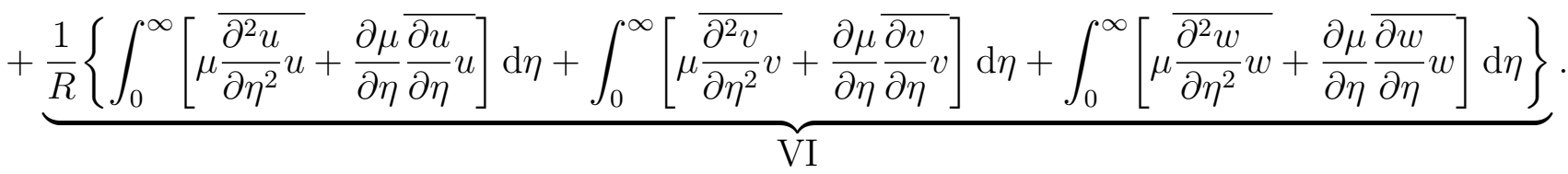

Note that overbars denote a period-averaged quantity; for example, $\overline{u v}=u v^{*}+u^{*} v$ (where * indicates a complex conjugate).

The energy equation can be normalized for any eigenmode against the integrated mechanical energy flux in the following form

$$
\begin{aligned}
-2 \bar{\alpha}_{\mathrm{i}} & =\underbrace{\left(P_{1}+P_{2}+P_{3}\right)}_{I}+\underbrace{D}_{I I}+\underbrace{\left(P W_{1}+P W_{2}\right)}_{I I I}+\underbrace{\left(S_{1}+S_{2}+S_{3}\right)}_{I V} \\
& +\underbrace{\left(G_{1}+G_{2}+G_{3}\right)}_{V}+\underbrace{\left(N_{1}+N_{2}+N_{3}\right)}_{V I} .
\end{aligned}
$$

Note that the mathematical origin of each term is indicated by the numbered underbracing in Eqs. (18) and (19).

As discussed by Cooper and Carpenter [28], Cooper et al. [29] and Garrett et al. [30], particular terms in equation (19) can be interpreted physically as originating from the following effects:

(I) the Reynolds stress energy production,

(II) the viscous dissipation energy removal term,

(III) pressure work terms,

(IV) contributions from work done on the wall by viscous stresses,

(V) terms arising from the streamline curvature effects and the three dimensionality of the mean flow, 
(VI) non-Newtonian viscosity terms.

The positive terms in the (19) contribute to energy production while the negative terms remove energy from the disturbances. A mode is therefore amplified when energy production outweighs the energy dissipation. That is, we have instability when $\alpha_{i}<0$, which is consistent with the definition of instability used to generate the neutral curves in Section 3.

Figure 4 shows the energy balance calculations for $R o=-1,-0.5$ and 0 computed on the maximum amplified type I mode at $R=R_{c}+25$. That is, those modes identified with red dots in Figure 3. The results confirm the stabilising effect on the type I mode for shear-thinning power-law fluids. This is due to a general reduction in the energy of the system. In each case the Reynolds stress $\left(P_{2}\right)$ and conventional viscous dissipation $(D)$ are the main contributors to the total energy, while the viscous dissipation energy removal term contributes to reduction of the total energy. It is clear that each of these effects is reduced as $n$ decreases. Figure 4 demonstrates that the non-Newtonian viscosity terms, $N_{1}, N_{2}$ and $N_{3}$, have only a very slight effect on the type I instability mechanism. That is, although we have identified shear-thinning effects to be universally stabilising, this is due to the modification of the steady flows and their subsequent beneficial interaction with the inviscid instability mechanism. The calculation of the energy balance for the Bödewadt flow is not applied here because of the very early onset of absolute instability, as was discussed previously. 


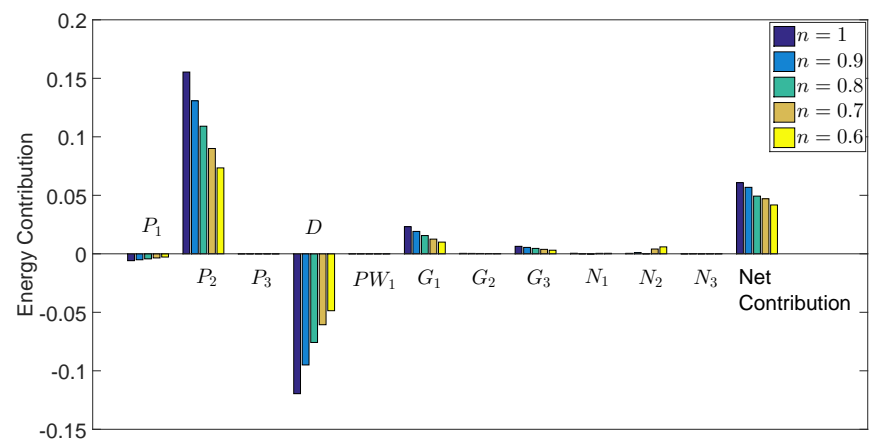

(a) von Kármán, $R o=-1$

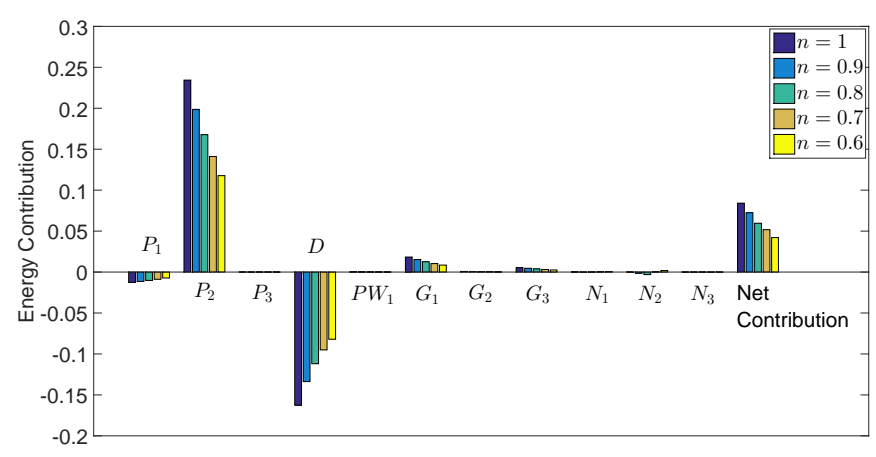

(b) $R o=-0.5$

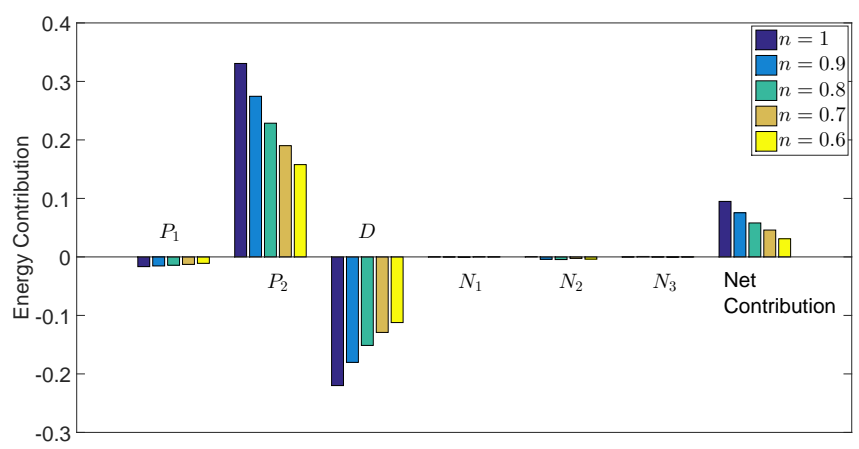

(c) Ekman, $R o=0$

Figure 4: Type I energy balance at $R e=R_{c}+25$ for Newtonian and shear-thinning power-law fluids with $n=1,0.9,0.8,0.7,0.6$. 


\section{Conclusions}

We have investigated the stability of stationary convective disturbances in the BEK family of boundary-layer flows for shear-thinning power-law fluids. We have successfully generalised the work of Griffiths et al. [23] to this broader class of flows and also allowed for a number of mathematical terms that had been previously neglected in that analysis. As with Griffiths' study, the analysis has required the use of the so-called parallel-flow approximation. Although this may lead to some slight inaccuracies in our qualitative predictions, the use of this approximation is common in the community and is not expected to affect the quantitative conclusions made here.

Our newly-derived steady-flow solutions are perturbed and a linear stability analysis has been conducted. Both sets of governing equations are controlled by the power-law index (defining the extent of shear thinning) and the Rossby number (defining the global rotation rate). The results presented show that shear-thinning fluids are expected to be stabilising for the BEK family of flows. In particular, we have shown that there is a stabilising effect on both the type I and type II modes. This is evident through a delayed onset of convective instability to higher Reynolds numbers and also qualitatively 'weaker' modes.

Our results have been confirmed by energy-balance calculations in order to obtain the underlying physical mechanisms behind the stabilising effects. This approach suggests that all physical processes (both energy production and dissipation) are weakened by the introduction of shear-thinning fluids. Furthermore, non-Newtonian viscosity is expected to play very little role in the dominant instability mechanisms. Rather our results suggest that their benefit comes from a modification of the steady flow profiles which, in turn, are more stable to inviscid type I effects.

With regards to our motivating industrial aims, we conclude that skin-friction drag in enclosed rotor-stator devices can be reduced through the use of shear-thinning lubricants. This would work by delaying laminar-turbulent transition, thereby utilising the lower drag forces arising from laminar flow.

There are several areas of research for future work on the current study. The results presented here could be extended to shear-thickening fluids and also alternative non-Newtonian viscosity models. Furthermore, this current study has been limited to stationary disturbances that are expected to be observed in all practical engineering applications where they are continuously excited by unavoidable surface roughness . However, where great care is taken to use high polished surfaces, it is known that non-stationary convective instabilities can become 
dominant in the sense of both larger growth rates and lower critical Reynolds numbers [31]. An allowance for non-stationary modes would then necessarily lead to the onset of absolute instability which is also a potential area of future interest.

\section{Acknowledgments}

MAA and BA wish to respectively recognise financial support from the Higher Committee for Education Development in Iraq (HCED), Republic of Iraq and the Ministry of National Education, Republic of Turkey. SJG is supported by a Senior Research Fellowship of the Royal Academy of Engineering, funded by the Leverhulme Trust.

\section{References}

[1] T. von Kármán, Über laminare und turbulente Reibung, Z. Angew. Math. Mech. 1 (1921) $233-252$.

[2] N. Gregory, J. T. Stuart, W. S. Walker, On the stability of three-dimensional boundary layers with applications to the flow due to a rotating disk, Phil. Trans. R. Soc. Lond. A 248 (1955) 155-199.

[3] M. R. Malik, The neutral curve for stationary disturbances in rotating-disk flow, J. Fluid Mech. 164 (1986) 275-287.

[4] P. Hall, An asymptotic investigation of the stationary modes of instability of the boundary layer on a rotating disc, Proc. R. Soc. Lond. A 406 (1986) 93-106.

[5] R. J. Lingwood, Absolute instability of the boundary layer on a rotating disk, J. Fluid Mech. 299 (1995) 17-33.

[6] C. Davies, P. W. Carpenter, Global behaviour corresponding to the absolute instability of the rotating-disk boundary layer, J. Fluid Mech. 486 (2003) 287-329.

[7] B. Pier, Finite-amplitude crossflow vortices, secondary instability and transition in the rotating-disk boundary layer, J. Fluid Mech. 487 (2003) 315-343.

[8] E. Appelquist, P. Schlatter, P. H. Alfredsson, R. J. Lingwood, Global linear instability of the rotating-disk flow investigated through simulations, J. Fluid Mech. 765 (2015) 612-631.

[9] V. W. Ekman, On the Influence of the Earth's Rotation on Ocean Currents, Ark. Math. Astron. Fysik. (1905). 
[10] U. T. Bödewadt, Die Drehströmung über festem Grund, Z. Angew. Math. Mech. 20 (1940) $241-252$.

[11] G. K. Batchelor, Note on the class of solutions of the Navier-Stokes equations representing steady non-rotationally symmetric flow, Q. J. Mech. Appl. Math. 4 (1951) 29-41.

[12] M. H. Rogers, G. N. Lance, The rotationally symmetric flow of a viscous fluid in the presence of an infinite rotating disk, J. Fluid Mech. 7 (1960) 617-631.

[13] A. J. Faller, Instability and transition of disturbed flow over a rotating disk, J. Fluid Mech. 230 (1991) 245-269.

[14] R. J. Lingwood, Absolute instability of the Ekman layer and related rotating flows, J. Fluid Mech. 331 (1997) 405-428.

[15] R. J. Lingwood, S. J. Garrett, The effects of surface mass flux on the instability of the BEK system of rotating boundary-layer flows, Euro. J. Mech. (B/Fluids) 30 (2011) 299-310.

[16] R. J. Lingwood, An experimental study of absolute instability of the rotating-disk boundary-layer flow, J. Fluid Mech. 314 (1996) 373-405.

[17] S. Imayama, R. J. Lingwood, P. H. Alfredsson, The turbulent rotating-disk boundary layer, Euro. J. Mech. (B/Fluids) 48 (2014) 245-253.

[18] S. Imayama, P. H. Alfredsson, R. J. Lingwood, Experimental study of rotating-disk boundary-layer flow with surface roughness, J. Fluid Mech. 786 (2016) 5-28.

[19] P. Mitschka, J. Ulbrecht, Nicht-Newtonsche Flüssigkeiten IV. Strömung Nicht-newtonsche Flüssigkeiten Ostwald-de Waelescher Typs in der Umgebung Rotierender Drehkegel und S chieben, Coll. Czech. Chem. Comm. 30 (1965) 2511-2526.

[20] H. I. Andersson, E. de Korte, R. Meland, Flow of a power-law fluid over a rotating disk revisited, Fluid Dyn. Res. 28 (2001) 75-88.

[21] J. P. Denier, R. E. Hewitt, Asymptotic matching constraints for a boundary-layer flow of a power-law fluid, J. Fluid Mech. 518 (2004) 261-279.

[22] P. T. Griffiths, S. O. Stephen, A. P. Bassom, S. J. Garrett, Stability of the boundary layer on a rotating disk for power law fluids, J. Non-Newtonian Fluid Mech. 207 (2014) 1-6. 
[23] P. T. Griffiths, S. J. Garrett, S. O. Stephen, The neutral curve for stationary disturbances in rotating disk flow for power-law fluids, J. Non-Newtonian Fluid Mech. 213 (2014) 73-81.

[24] P. T. Griffiths, Flow of a generalised Newtonian fluid due to a rotating disk, J. NonNewtonian Fluid Mech. 221 (2015) 9-17.

[25] E. C. Bingham, An investigation of the laws of plastic flow, Bull. U. S. Bur. of Standards 13 (1916) 309-352.

[26] P. J. Carreau, Rheological Equations from Molecular Network Theories, Trans. Soc. Rheolo. 16:1 (1972) 99-127.

[27] B. Alveroglu, A. Segalini, S. J. Garrett, The effect of surface roughness on the convective instability of the BEK family of boundary-layer flows, Euro. J. Mech. (B/Fluids) 56 (2016) $178-187$.

[28] A. J. Cooper, P. W. Carpenter, The stability of rotating-disc boundary-layer flow over a compliant wall. Part 1. Type i and ii instabilities, J. Fluid Mech. 350 (1997) 231-259.

[29] A. J. Cooper, J. H. Harris, S. J. Garrett, M. Özkan, P. J. Thomas, The effect of anisotropic and isotropic roughness on the convective stability of the rotating disk boundary layer, Phys. Fluids 27 (2015) 014107.

[30] S. J. Garrett, A. J. Cooper, J. H. Harris, M. Özkan, A. Segalini, P. J. Thomas, On the stability of von Kármán rotating-disk boundary layers with radial anisotropic surface roughness, Phys. Fluids 28 (2016) 014104.

[31] P. Balakumar, M. R. Malik, Traveling disturbances in rotating-disk flow, Theor. Comput. Fluid Dyn. 2 (1990) 125-137. 